\title{
Status of SuperKEKB phase-2 commissioning
}

\author{
Akio Morita* for SuperKEKB commissioning group \\ KEK \\ E-mail: akio.morita@kek.jp
}

\begin{abstract}
SuperKEKB is an asymmetric energy electron-positron collider for Belle II experiment. It is designed to achieve 40 times luminosity of the previous KEKB B-Factory accelerator by using the novel "nano-beam" collision scheme. The SuperKEKB accelerator commissioning is devided in 3 stages. The first stage so called "phase-1 commissioning" has been performed in 2016 for testing the accelerator system without the final focus system and for verifying the low emittance beam tuning. After installation of the final focus system and the Belle II detector, the second stage so called "phase-2 commissioning" has started from 19th March 2018 to verify the "nano-beam" collision. We report the preliminary result of the phase- 2 commissioning.
\end{abstract}

The 39th International Conference on High Energy Physics (ICHEP2018)

4-11 July, 2018

Seoul, Korea

${ }^{*}$ Speaker. 


\section{Introduction}

SuperKEKB is a double ring collider for Belle II experiment, which is made of $7 \mathrm{GeV}$ electron high energy ring(HER) and $4 \mathrm{GeV}$ positron low energy ring(LER). Its design luminosity is $8 \times$ $10^{35} \mathrm{~cm}^{-2} \mathrm{~s}^{-1}$ which is 40 times the performance of the previous KEKB B-Factory accelerator. In order to achieve such high luminosity, the design vertical beta function at the interaction point(IP) is squeezed smaller than the design bunch length. However, traditional collision scheme in this parameter region does not work fine. For avoiding this issue, a kind of large Piwinski angle collision scheme so called "nano-beam" is introduced.

The SuperKEKB commissioning is divided into 3 stages: phase-1, phase- 2 and phase-3. Before installing both the final focus system and the Belle II detector, the phase-1 commissioning has been performed from 1st February 2016 to 28th June 2016 for testing the storage ring hardware except the final focus system and for establishing the low emittance beam operation. After the phase- 1 commissioning both the final focus system and the Belle II detector were installed. The phase-2 commissioning is performed from 19th March 2018 to 17th July 2018 for verifying the "nano-beam" collision scheme.

\section{Nano-beam collision scheme}

In the SuperKEKB design[1], the vertical beta function at the IP is designed as $300 \mu \mathrm{m}$, which is one twentieth of the design bunch length $6 \mathrm{~mm}$. A beta function around the IP describes as

$$
\beta(s)=\beta^{*}\left(1+\left(\frac{s}{\beta^{*}}\right)^{2}\right),
$$

where $\beta^{*}$ is a beta function at the IP. In the case that $\beta_{y}^{*}$ is smaller than a bunch length $\sigma_{z}$, the off waist beam collision luminosity is degraded because of the increased vertical beam size due to Eq. 2.1 so called the hourglass effect. Therefore, a beam-beam interaction between different vertical beam sizes due to the hourglass effect makes an instability and blocks a high beam-beam parameter collision. The SuperKEKB "nano-beam" scheme avoids the hourglass effect by introducing a large horizontal beam crossing angle to separate off waist beams. In the case that a horizontal displacement $\theta_{h c} \sigma_{z}$ is enough larger than a horizontal beam size $\sigma_{x}^{*}$, a effective colliding bunch length $\sigma_{z}^{e f f}$ is approximated as $\sigma_{x}^{*} / \theta_{h c}$, where $\theta_{h c}$ is a half crossing angle. In order to avoid serious consequences from the hourglass effect, the SuperKEKB design parameters are chosen to satisfy $\beta_{y}^{*} \sim \sigma_{z}^{e f f} \sim \sigma_{z} / 20$. Under the assumption that the colliding vertical beam sizes are equivalent, the "nano-beam" scheme luminosity is approximated as

$$
L \sim \frac{\gamma_{ \pm}}{2 e r_{e}} \frac{I_{ \pm} \xi_{y \pm}}{\beta_{y}^{*}}
$$

where $\gamma_{ \pm}, I_{ \pm}$and $\xi_{ \pm}$are Lorentz gamma factor, beam current and vertical beam-beam parameter of colliding beams, respectively. The beam-beam parameter in Eq. 2.2 is described as

$$
\xi_{ \pm}=\frac{r_{e} N_{\mp}}{2 \pi \gamma_{ \pm} \sigma_{x}^{* e f f}} \sqrt{\frac{\beta_{y}^{*}}{\varepsilon_{y}}},
$$


where $N_{\mp}, \sigma_{x}^{* e f f}$ and $\varepsilon_{y}$ are a number of particles in colliding bunches, an effective horizontal beam size $\left.\sqrt{\sigma_{x}^{* 2}+\left(\theta_{h c} \sigma_{z}\right.}\right)^{2}$ and a vertical beam emittance of colliding beams, respectively. In the "nano-beam" scheme, $\sigma_{x}^{* e f f}$ is approximated as $\theta_{h c} \sigma_{z}$. Thus the luminosity and the beam-beam parameter do not depend with the IP horizontal beta function $\beta_{x}^{*}$. Equation 2.2-2.3 predict that the luminosity is improved in inverse proportion to the square root of $\beta_{y}^{*}$ if $\varepsilon_{y}$ is kept during $\beta_{y}^{*}$ squeezing. In order to keep the beam-beam parameter, $\varepsilon_{y}$ must be reduced in proportion to $\beta_{y}^{*}$.

\section{Phase-2 commissioning}

The major purposes of the phase- 2 commissioning are the verification of the nano-beam collision scheme and to study the beam collision property and the detector background property for the next phase- 3 commissioning. As the first step of the phase- 2 commissioning, we stored beams on the detuned optics, whose $\beta_{y}^{*}$ are $48.6 \mathrm{~mm}$ for LER and $81 \mathrm{~mm}$ for HER, and verified the functionality of the final focus system and establish the global orbit \& optics correction. After the establishment of the beam storage, the check and adjustment of the accelerator system components: magnet control, RF, monitoring system and feedback system were performed. In parallel with these tasks, the vacuum scrabbling was performed to improve vacuum pressure. After such preconditioning, IP beta functions were squeezed for the first collision test. The first beam collision was achieved by adjusting the relative RF phase and the IP orbit between two beams using the beam-beam deflection and the Belle II luminosity monitor. These early phase- 2 commissioning details until the first collision is reported on the IPAC 2018 proceedings[2]. After the collision establishment, the following step-by-step $\beta_{y}^{*}$ squeezing and the luminosity tuning were performed for verifying the nano-beam collision scheme.

\section{1 $\beta_{y}^{*}$ squeezing}

In the first $\beta_{y}^{*}$ squeezing trial at early April, we tried to squeeze HER $\beta_{y}^{*}$ down to $2.4 \mathrm{~mm}$ directly. However, the beam storage trials on the squeezed collision optics failed due to the final focus superconducting quadrupole(QCS) magnet quenches. Therefore, for avoiding operation difficulty due to such QCS magnet quenches, the IP beta functions for the first collision test were chosen as $200 \mathrm{~mm}$ for $\beta_{x}^{*}$ and $8 \mathrm{~mm}$ for $\beta_{y}^{*}$, respectively. After some investigations, it was understood that these QCS magnet quenches were caused by an injection beam loss around the vertical final focus superconducting quadrupole(QC1) magnet due to the enlarged vertical beta function at QC1 magnet and the XY-coupling error enhanced by the $\beta_{y}^{*}$ squeezing. In order to protect QCS magnets from such beam loss, we closed the beam collimators which ware installed to protect the Belle II detector from the beam background by cutting the particles exceeded QCS magnet aperture and connected the fast detector background monitors which were sensitive to beam loss around QCS magnets to the beam abort system in order to abort stored beam before QCS magnet quench due to beam loss. In order to reduce the optics error after $\beta_{y}^{*}$ squeezing, we reduced the step size of $\beta_{y}^{*}$ squeezing and performed the global optics correction [3, 4], injection tuning and beam collimator tuning to prevent the enhancement of the optics error by the next squeezing step. Table 1 shows the history of the typical operated optics by this step-by-step $\beta_{y}^{*}$ squeezing approach, where $L_{\text {peak }}$ is the peak luminosity under the controlled beam condition denoted by $I_{L E R}, I_{H E R}$ and $n_{b}$ (number of bunches) for luminosity performance comparisons. The "Phase" in Table 1 denotes the set of 


\begin{tabular}{|c|c|c|c|c|c|} 
Phase & $\beta_{x}^{*}[\mathrm{~mm}]$ & $\beta_{y}^{*}[\mathrm{~mm}]$ & Begin & $L_{\text {peak }}\left[\mathrm{cm}^{-2} \mathrm{~s}^{-1}\right]$ & $I_{L E R} / I_{H E R}[\mathrm{~mA}], n_{b}$ \\
& LER / HER & LER / HER & & & \\
2.1 .0 & 200 & 8 & $2018-04-16$ & $0.93 \times 10^{33}$ & $250 / 220,600$ \\
2.1 .1 & 200 & 6 & $2018-05-22$ & $1.37 \times 10^{33}$ & $340 / 285,789$ \\
2.1 .2 & 200 & 4 & $2018-05-28$ & $1.36 \times 10^{33}$ & $340 / 285,789$ \\
2.1 .3 & 200 & $4 / 3$ & $2018-06-08$ & $1.32 \times 10^{33}$ & $340 / 285,789$ \\
2.1 .4 & 200 & 3 & $2018-06-11$ & $1.05 \times 10^{33}$ & $320 / 265,789$ \\
2.1 .5 & 100 & 4 & $2018-06-12$ & $1.09 \times 10^{33}$ & $340 / 285,789$ \\
2.1 .6 & $200 / 100$ & 4 & $2018-06-13$ & $2.04 \times 10^{33}$ & $350 / 295,789$ \\
2.1 .7 & $200 / 100$ & 3 & $2018-06-20$ & $2.60 \times 10^{33}$ & $340 / 285,789$ \\
2.2 .0 & 200 & 2 & $2018-06-07$ & na & $50 / 50,1576$ \\
2.3 .1 & na / 100 & na / 1.5 & $2018-07-09$ & na & na/50, 1576
\end{tabular}

Table 1: Summary of Beta Squeezing

the IP beta functions. The minimum achieved $\beta_{y}^{*}$ are $2 \mathrm{~mm}$ for LER(Phase 2.2.0) and $1.5 \mathrm{~mm}$ for HER(Phase 2.3.1), however, these are operated for the optics correction study only. The minimum $\beta_{y}^{*}$ for the collision operation is $3 \mathrm{~mm}$ shown as Phase 2.1.7 in Table 1.

\subsection{Luminosity tuning}

During collision operation, beam collision was maintained with the slow IP vertical orbit feedback software to keep the vertical beam-beam deflection measured by the QCS BPMs. The dithering feedback system for IP horizontal collision offset had been tested during the phase- 2 commissioning, however, it was not used for the daily operation because the phase- 2 horizontal collision is enough stable. This dithering feedback system will be used in the phase-3 operation. In beam decaying operation for physics run of the Belle II detector, colliding beam state depends with the beam current. The comparison of specific luminosity, which is a luminosity performance normalized by bunch current product, between different tuning parameter sets does not work well, because colliding bunch current ratio is changed due to different beam lifetime between HER and LER. In order to stabilize the colliding beam condition during luminosity tuning, a top-up injection was performed. The Belle II detector data acquisition was stopped during such luminosity tuning operation, because data acquisition under the top-up injection was not confirmed by the Belle II detector system at this moment. In the other hand, both luminosity monitors and beam background detectors were working and referenced for the luminosity tuning.

In each $\beta_{y}^{*}$ squeezing steps, the following IP optical parameter tuning knobs are scanned to maximize the measured specific luminosity. During such knob tuning, the detector background detectors, the beam size monitors and the beam lifetime are monitored to avoid collision condition degradation. As the first step for the luminosity tuning study, the vertical collision offset for the collision orbit feedback system is adjusted, because the best offset for maximizing luminosity is changed due to the machine drift. For major luminosity tuning, both the XY-coupling parameters $R 1^{*}, R 2^{*}, R 3^{*}$ and $R 4^{*}$ and the vertical dispersion parameters $\eta_{y}^{*}$ and $\eta_{y}^{\prime *}$ are scanned by the linear perturbation knob constructed by both standalone skew quadrupole corrector magnets in the interaction region and skew quadrupole corrector windings on sextupole magnets for chromaticity 

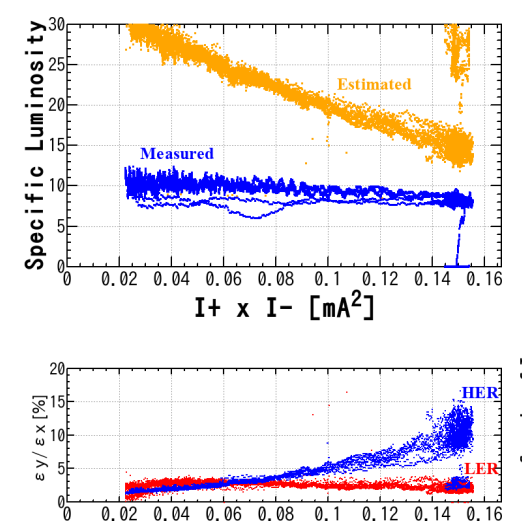

(a) Specific luminosity before IP local coupling adjustment
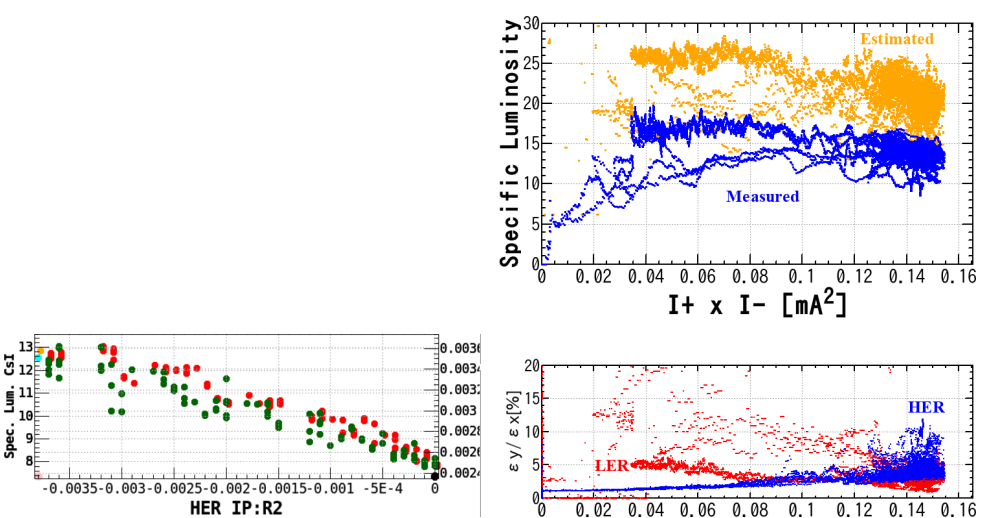

(b) HER $R 2^{*}$ scan result

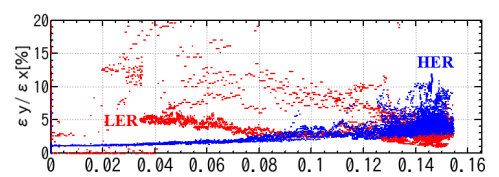

(c) Specific luminosity after IP local coupling adjustment

Figure 1: Specific luminosity: Figure 1a and 1c show the bunch current product dependency of the specific luminosity in the different collision conditions on the phase 2.1.6 optics before and after the IP local coupling adjustment, respectively. In upper charts of Fig.1a and 1c, the blue "measured" line shows the measured specific luminosity calculated from the luminosity and the bunch currents. The yellow "estimated" line shows the specific luminosity expected from the measured beam size by using an analytic formula. The lower charts of Fig.1a and 1c show the vertical beam sizes of two rings measured by the X-ray beam size monitors as the emittance coupling ratio. Figure $1 \mathrm{~b}$ shows the specific luminosity in the HER XY-coupling parameter $R 2^{*}$ scan.

correction, where $R 1, R 2, R 3$ and $R 4$ are coupling parameters in SAD notation[5]. In addition, the vertical waist, which is the longitudinal position of the focal point of the vertical optics, is scanned by using the QC1 quadrupole magnets.

\subsection{Luminosity scaling issue and IP local coupling}

At the first $\beta_{y}^{*}$ squeezing from $6 \mathrm{~mm}$ to $4 \mathrm{~mm}$ corresponding with Phase 2.1.1 to 2.1.2 transition in Table 1, we did not observe luminosity improvement unlike the predication of Eq.2.2-2.3. The vertical emittance estimated from the X-ray beam size monitor installed at the end of the arc section was kept after this $\beta_{y}^{*}$ squeezing. No significant error was found in the measured beta function, $\mathrm{XY}$-coupling and dispersion function during the global optics correction. These measurements suggest $\beta_{y}^{*}$ squeezing success and vertical emittance conservation. In the other hand, the effective IP vertical beam size measured by the vertical collision offset scan at an ultra low bunch current operation is $1.2 \mu \mathrm{m}$ assuming that vertical colliding beam sizes are equivalent. However, the vertical IP beam sizes estimated from the X-ray beam size monitors are $0.4 \mu \mathrm{m}$ for LER and $0.5 \mu \mathrm{m}$ for HER, respectively. This beam size discrepancy suggests a geometrical mismatch of colliding beam profiles at the IP due to a local optical function error. This hypothesis is supported by almost constant specific luminosity during a beam size change due to a bunch current decay shown in Fig. 1a. In the other words, the effective IP vertical beam size does not depend with the individual beam sizes measured by the X-ray beam size monitors. In order to identify the luminosity degradation source, the vertical beam waist position and the IP XY-coupling/dispersion parameters $\left(R 1^{*}, R 2^{*}, R 3^{*}\right.$, 
$R 4^{*}, \eta_{y}^{*}$ and $\eta_{y}^{\prime *}$ ) were scanned by using the luminosity tuning knobs. From these IP parameter scans, we found a big $R 2^{*}$ error in HER shown in Fig. 1b. After some investigation, this local $R 2^{*}$ error was adjusted by using the skew quadrupole correctors of the QC1 quadrupoles, because the total amount of detected $R 2^{*}$ error $6 \mathrm{~mm}$ was too big to adjust by the perturbative IP tuning knob. After such IP local coupling adjustments, the luminosity of the corrected $\beta_{y}^{*}=3 \mathrm{~mm}$ optics shown in Table 1 as Phase 2.1.6 was improved up on $1 / \sqrt{\beta_{y}^{*}}$ scaling line and the effective vertical beam size measured by the offset scan became $0.7 \mu \mathrm{m}$ and the specific luminosity is improved as shown in Fig. 1c. And, furthermore luminosity scaling was confirmed until $\beta_{y}^{*}=3 \mathrm{~mm}$ collision optics.

\section{Summary of Phase-2 Commissioning Achievements}

The achieved maximum stored beam currents are $858 \mathrm{~mA}$ for LER and $788 \mathrm{~mA}$ for HER, respectively. The LER current has be limited by the longitudinal coupled bunch instability This instability source is not understood clearly, however, it it turned out that RF cavities are not an instability source from the measured instability mode spectrum and some study results suggest the relationship between collimator aperture settings and this instability strength. This LER instability will be suppressed by activating the longitudinal bunch-by-bunch feedback system and furthermore study will be performed in the next phase-3 commissioning. In the other hand, the HER maximum current has be limited by the study time of the high current operation. No issue has be found to limit the HER current at this moment. The luminosity achievements in the $\beta_{y}^{*}=3 \mathrm{~mm}$ collision optics is summarized as follows. The maximum luminosity is $5.55 \times 10^{33} \mathrm{~m}^{-2} \mathrm{~s}^{-1}$ which is recorded in 1576 bunch operation with $788 / 778 \mathrm{~mA}(\mathrm{LER} / \mathrm{HER})$ stored beam current. The maximum specific luminosity in the high bunch current operation is $1.57 \times 10^{31} \mathrm{~m}^{-2} \mathrm{~s}^{-1} / \mathrm{mA}^{2}$ which is recorded in 395 bunch operation with $265 / 217 \mathrm{~mA}$ (LER/HER) stored beam current and the achieved beam-beam parameter of the weak beam $\xi_{y-}$ is 0.021 in the same condition. The extrapolation to 1576 bunch operation expects $9.1 \times 10^{33} \mathrm{~m}^{-2} \mathrm{~s}^{-1}$ luminosity with $1057 / 865 \mathrm{~mA}(\mathrm{LER} / \mathrm{HER})$ stored beam current, however, it is not achieved due to the instability issue. In this phase- 2 commissioning, we achieved the stable collision operation with $\beta_{y}^{*}=3 \mathrm{~mm}$ collision optics and confirmed that the specific luminosity is scaled by the inverse square root $\beta_{y}^{*}$. The "nano-beam" collision scheme looks like to work fine with $\beta_{y}^{*}$ squeezed until the half of the bunch length. The further lower $\beta_{y}^{*}$ collision would be tried in the next phase- 3 commissioning. The phase- 3 commissioning dedicated for the physics run would be planned to start in March 2019 after the pixel vertex detector installation.

\section{References}

[1] Y. Ohnishi et al, Accelerator design at SuperKEKB, Prog. Theor. Exp. Phys. 2013 03A011 (2013)

[2] Y. Ohnishi, Report on SuperKEKB Phase 2 Commissioning, Proc. of IPAC2018, Vancouver, BC, Canada, April 2018, MOXGB1, p. 1 (2018)

[3] Y. Ohnishi et al, Optics Measurements and Corrections at the Early Commissioning of SuperKEKB, Proc. of IPAC2016, Busan, Korea, May 2016, THPOR007, p. 3782 (2016)

[4] Y. Ohnishi et al, Optics Correction and Low Emittance Tuning at the Phase 1 Commissioning of SuperKEKB, Proc. of eeFACT2016, Daresbury, UK, October 2016, TUT3BH2, p. 143 (2016)

[5] x-y-coupling parameter definition of SAD, http://acc-physics.kek.jp/SAD/SADHelp.html\#L539 